\title{
Near-infrared-active upconverting niobium-doped black titanium dioxide photocatalysts
}

\author{
Collins Nganou, ${ }^{a}$ Andrew J. Carrier, ${ }^{a}$ Ali Shafiee, ${ }^{a}$ Naizhen Yu, ${ }^{a}$ Ken D. Oakes, ${ }^{b}$ and Xu Zhang ${ }^{a *}$ \\ aDepartment of Chemistry, Cape Breton University, 1250 Grand Lake Road, Sydney, Nova Scotia, Canada, \\ B1P 6L2

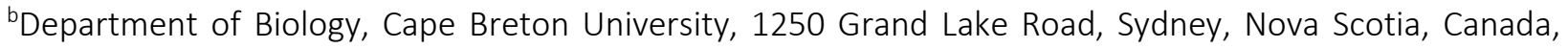 \\ B1P 6L2 \\ *Corresponding author. E-mail: Xu_Zhang@cbu.ca
}

\begin{abstract}
Black titanium dioxide is a broadband-absorbing photocatalyst characterized by integration of atomic hydrogen within the outer layers of anatase nanomaterials. In addition to is broad absorbance of visible to near-infrared light, the material has some intrinsic upconversion ability, which is greatly enhanced by the addition of a niobium oxide shell. This yields a highly active photocatalyst as demonstrated in photodegradation experiments.
\end{abstract}

\section{Introduction}

Anthropogenic water pollution is a persistent problem for aquatic environmental health and clean water accessibility in developed and developing communities. Photocatalytic degradation of organic pollutants is an attractive water treatment method because it can be performed passively through the action of sunlight. However, the most prominent and well-studied photocatalytic material, titanium dioxide, is limited in that its bandgap can only be excited by ultraviolet (UV) light, which only represents a small portion of the entire solar spectrum. Electron-hole pairs generated on the $\mathrm{TiO}_{2}$ surface through UV excitation have high energy, which degrade pollutant molecules, e.g., through the formation of hydroxyl radicals; however, $\mathrm{TiO}_{2}$ suffers rapid charge recombination and consequently poor photo-efficiency. Rapid charge recombination can be addressed through the formation of composite materials, but poor solar spectrum utilization is not easily solved.

Upconversion nanomaterials are those that absorb lower-energy photons, i.e., visible and near-infrared (NIR) radiation, which are subsequently pumped to populate higher energy states. The presence of dopants in the titanium dioxide crystal structure can modify its electronic band structure, with the presence of hydrogen in the outer layers of anatase generating black titanium dioxide $\left(\mathrm{bTiO}_{2}\right)$, which has absorbance in the visible and NIR spectrum. Herein we generated black titanium dioxide and integrated of niobium oxides in the outer layers, demonstrated its photocatalytic efficiency, and decoupled the effects of $\mathrm{NbO}_{2}$ and $\mathrm{Nb}_{2} \mathrm{O}_{5}$ phases on charge mobility and energy transfer. We demonstrated the intrinsic upconversion ability of $\mathrm{bTiO}_{2}$, which is greatly enhanced by the addition of niobium oxides to the outer layers.

\section{Experimental}

\section{Materials}

Titanium (IV) isopropoxide and Rhodamine B were obtained from Sigma Aldrich (Oakville, ON, Canada). Niobium n-butoxide was obtained from Fisher Scientific (Ottawa, ON, Canada). All materials were of reagent grade or higher and were used without further purification. 


\section{Instruments}

Powder X-ray diffraction (XRD) experiments were performed using an Advance D8 X-ray diffractometer (Brucker, Billerica, MA, USA) using CuKa $(\lambda=1.5406 \AA$ ) radiation. Transmission electron microscopy (TEM) experiments were performed using an HT7700 transmission electron microscope (Hitachi, Tokyo, Japan) with an acceleration potential of $80 \mathrm{kV}$. X-ray photoelectron spectroscopy (XPS) experiments were performed using an AXIS Nova spectrometer (Kratos Analytical, Manchester, UK) using an AI X-ray source and a detector with a work function of $4.49 \mathrm{eV}$. The samples were mounted onto a scanning electron microscope mount (with the pins cut off) using double-sided adhesive Cu tape and were held under high vacuum $\left(10^{-9} \mathrm{Torr}\right)$ overnight inside the preparation chamber before transferring into the analysis chamber (ultrahigh vacuum, $10^{-10}$ Torr) of the spectrometer. Electron spin resonance (ESR) experiments were performed using a microESR spectrometer (Bruker, Billerica, MA, USA) with a microwave power and digital gain of $15 \mathrm{~mW}$ and $12 \mathrm{~dB}$, respectively. Nitrogen physisorption analysis was performed using an ASAP 2020 analyzer (Micromeritics, Norcross, GA, USA). Zeta potential measurements were performed on a Nanosizer (Horiba, Kyoto, Japan). Solar radiation was simulated with a 11002 Sunlite simulator (ABET Technologies, Milford, CT, USA) using a Xe arc lamp. NIR light was applied using a diode laser ( $808 \mathrm{~nm}, 2 \mathrm{~W}$, Chengchun New Industries Optoelectronics Tech. Co. Ltd., Chengchun, China). Total organic carbon (TOC) was measured using a MULTI N/C UV HS analyzer (Analytikjena, Jena, Germany). UV-Vis spectra were obtained using an Infinite M1000 PRO plate reader (Tecan Group Ltd., Männedorf, Zürich, Switzerland) using Costar 96-well flat-bottom transparent polystyrol microplates (Corning, NY, USA).

$\mathrm{Nb}_{x} \mathrm{O}_{y}-\mathrm{TiO}_{2}$ core-shell nanoparticles

Titanium (IV) isopropoxide (15 mL, $50 \mathrm{mM}$ ) and niobium (V) n-butoxide $(5 \mathrm{~mL}, 10 \mathrm{mM})$ were dissolved in isopropanol $(15 \mathrm{~mL})$ and stirred for $3 \mathrm{~h}$ at $1200 \mathrm{rpm}$. Then, hydrazine hydrate $(1 \mathrm{~mL})$ was added dropwise to obtain a white gel that was transferred to a Teflon-lined hydrothermal reactor, which was subsequently sealed and heated to $250 \stackrel{\circ}{ } \mathrm{C}$ for $3 \mathrm{~h}$. The reactor was allowed to cool to ambient temperature before the mixture was transferred to a tube and centrifuged at $5000 \mathrm{rpm}$ for $15 \mathrm{~min}$ to isolate the particles from the supernatant, which were washed $x$ times using nanopure water before drying in an oven at 60 oC overnight.

\section{Black $\mathrm{Nb}_{x} \mathrm{O}_{y}-\mathrm{TiO}_{2}$ core-shell nanoparticles}

Hydrogen peroxide ( $1 \mathrm{~mL}, 30 \mathrm{wt} \%$ ) was added dropwise to the $\mathrm{Nb}_{x} \mathrm{O}_{y}-\mathrm{TiO}_{2}$ core-shell nanoparticles obtained above, which resulted in the nanoparticles turning yellow. The material was transferred to a vacuum oven

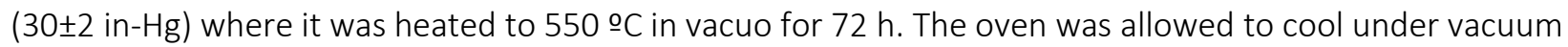
to ambient temperature before collecting the resultant black $\mathrm{Nb}_{\mathrm{x}} \mathrm{O}_{\mathrm{y}}-\mathrm{TiO}_{2}$ core-shell nanoparticles. Black $\mathrm{Nb}_{x} \mathrm{O}_{y}$ nanoparticles were prepared through an identical procedure using $\mathrm{Nb}_{x} \mathrm{O}_{\mathrm{y}}$ nanoparticles.

\section{$\mathrm{SiO}_{2}$-coated black $\mathrm{Nb}_{x} \mathrm{O}_{y}$ - $\mathrm{TiO}_{2}$ core-shell nanoparticles}

$\mathrm{SiO}_{2}$-coated black $\mathrm{Nb}_{x} \mathrm{O}_{y}-\mathrm{TiO}_{2}$ core-shell nanoparticles were prepared from equal volumes of the black $\mathrm{Nb}_{x} \mathrm{O}_{\mathrm{y}}-\mathrm{TiO}_{2}$ core-shell nanoparticles (1 wt\%) and tetraethylorthosilicate $(1 \mathrm{mM})$, which were ultrasonicated for $10 \mathrm{~min}$ in $10 \mathrm{~mL}$ of EtOH in a $15 \mathrm{~mL}$ polypropylene centrifuge tube. No aggregation of the resultant nanoparticles was observed after centrifugation at $12500 \mathrm{rpm}$ for $20 \mathrm{~min}$. The $\mathrm{SiO}_{2}$-coated black $\mathrm{Nb}_{\mathrm{x}} \mathrm{O}_{\mathrm{y}}-\mathrm{TiO}_{2}$ core-shell nanoparticles were washed 10 times with nanopure water.

\section{Computational modelling}


To compute the supercell, the selected area electron diffraction (SAED), and the powder ring diffraction patterns of the $\mathrm{Nb}_{x} \mathrm{O}_{y}-\mathrm{TiO}_{2}$ core-shell nanoparticles, the anatase and niobium oxide crystal structures were obtained from the American Mineralogist Crystal Structure Database (American Mineralogist, Volume 88, pages 247-250, 2003) and the material project database (doi:10.1063/1.4812323). The crystal structure of $\mathrm{TiO}_{2}$ anatase, $\mathrm{NbO}_{2}$, and $\mathrm{Nb}_{2} \mathrm{O}_{5}$ materials were optimized with $\mathrm{Jmol}$ and PM7 Hamiltonian of mopac2016. The $\mathrm{TiO}_{2}$ anatase, $\mathrm{NbO}_{2}$, and $\mathrm{Nb}_{2} \mathrm{O}_{5}$ diffraction patterns were compared with the crystal diffraction algorithm. Their SAED and powder ring diffraction patterns were modelled with the single crystal 4 . The calculated $\mathrm{TiO}_{2}$ anatase SAED was superimposed on the experimental SAED to identify the points associated with the anatase phase, whereas points deviating from the modelled $\mathrm{TiO}_{2}$ anatase SAED were used to identify the $\mathrm{NbO}_{2}$ and $\mathrm{Nb}_{2} \mathrm{O}_{5}$ patterns. The powder ring $\mathrm{TiO}_{2}$ anatase, $\mathrm{NbO}_{2}$, and $\mathrm{Nb}_{2} \mathrm{O}_{5}$ diffraction patterns were superimposed to corroborate the difference in the experimental SAED. The equivalent stereograph of the diffraction patterns was obtained from single crystal 4. CrystalMaker $X$ was used to generate the supercell of the $\mathrm{TiO}_{2}$ anatase, $\mathrm{NbO}_{2}$, and $\mathrm{Nb}_{2} \mathrm{O}_{5}$ composite.

\section{Photodegradation}

NIR-driven photocatalysis was performed by irradiating the sample $(0.42 \mathrm{wt} \%$ of photocatalyst with $50 \mu \mathrm{M}$ of sample, i.e., RhB or ABDA) with a NIR diode laser every $5 \mathrm{~min}$. During the irradiation, the sample was covered with aluminum to mitigate the effect of any scatter light originating from the computer screen in the darkroom. Blanks were performed in the dark and each reaction was performed in five replicates. Sunlight-driven photocatalysis was performed using SSL with a UV density 4 filter to remove UV photons.

\section{Reactive oxygen species analysis}

Reactive oxygen species (ROS) were detected indirectly through the application of specific scavengers. 4hydroxy-2,2,6,6-tetramethylpiperidine (TMP-OH) was used as an ROS probe that reacts with oxidants to generate the stable 4-hydroxy-2,2,6,6-tetramethylpiperidine oxyl radical (TEMPO-OH), which yields a diagnostic 1:1:1 triplet in its ESR spectrum. Each material was exposed to a solution of TMP-OH (250 mM) either in the light or dark in the presence of one of the following scavengers: t-butyl alcohol (500 mM), coumarin $(200 \mu \mathrm{M})$, sodium azide $(100 \mathrm{mM}), \mathrm{XTT}(100 \mu \mathrm{M})$, or a blank. The EPR spectra of each experiment were then recorded.

\section{Results and Discussion}

\section{Syntheses}

Black titanium dioxide and/or niobium oxides were formed through the dissociation and diffusion of atomic hydrogen from surface-bound hydrogen peroxide under heat and vacuum conditions. Displacement of water with surface-bound hydrogen peroxide on $\mathrm{Nb}$ surface sites immediately transforms the white anatase into a yellow material, which upon heating to 550 ㄷ C under vacuum yields a black material through the diffusion of atomic hydrogen generated through thermal homolytic bond cleavage and decomposition of the bound hydrogen peroxide $\left(B D E=460 \mathrm{~J} \mathrm{~mol}^{-1}\right)$ into the crystal lattice. The remaining oxygen is removed by the vacuum or remains adsorbed to the surface.

\section{Characterization}

Black $\mathrm{Nb}_{x} \mathrm{O}_{y}-\mathrm{TiO}_{2}$ consists of a mixed crystal structure phase, primarily anatase (space group 141/amd) arranged around distorted octahedra. $\mathrm{TiO}_{2}$ anatase forms the nanoparticle core, where epitaxial growth of 
$\mathrm{NbO}_{2}$ occurs on its surface at $550 \stackrel{\circ}{\mathrm{C}}$. Subsequent oxidation of the exterior surface to $\mathrm{Nb}_{2} \mathrm{O}_{5}$ generates a dual interface and prevents further oxidation of the middle $\mathrm{NbO}_{2}$ phase. The powder XRD pattern consists of anatase peaks with additional tetragonal $\mathrm{NbO}_{2}(2 \mathrm{O}-2)$ and several triclinic $\mathrm{Nb}_{2} \mathrm{O}_{5}$ reflection planes (Fig. 1). A rutile-like $\mathrm{NbO}_{2}$ phase is unlikely as it is only formed at $>1000 \mathrm{~K}$. The proposed dual $\mathrm{Nb}_{x} \mathrm{O}_{y}$ phases are supported by the O1s XPS spectrum (Fig. 2), which has peaks at 532.7 and $531.2 \mathrm{eV}$, corresponding to $\mathrm{Nb}_{2} \mathrm{O}_{5}$ and $\mathrm{NbO}_{2}$, respectively.

We postulate that tetragonal $\mathrm{NbO}_{2}$ grows adjacent to the bulk $\mathrm{TiO}_{2}$ anatase and triclinic $\mathrm{Nb}_{2} \mathrm{O}_{5}$ forms on the exterior. Black $\mathrm{Nb}_{x} \mathrm{O}_{y}-\mathrm{TiO}_{2}$ was modelled using a supercell structure with $\mathrm{TiO}_{2}$ anatase set at the nanostructure core (Fig. 3). The $\mathrm{NbO}_{2}$ phase shares oxygen atoms at the $\mathrm{TiO}_{2}$ interface whereas $\mathrm{Nb}_{2} \mathrm{O}_{5}$ shares oxygen atoms at its interface with $\mathrm{NbO}_{2}$. We used this model to predict the single-crystal electron diffraction pattern, which was superimposed onto the experimental selected area electron diffraction pattern (SAED, Fig. 4) with a good overlap between theoretical and experimental results.

The material consists of spheroidal nanoparticles (mean diameter $10 \mathrm{~nm}$, Fig. 5) encased in aggregated amorphous material. High-resolution TEM revealed 3.5 and $2.4 \AA$ lattice fringes in the anatase core and $\mathrm{Nb}_{x} \mathrm{O}_{y}$ shell, respectively, which is coated in an amorphous layer (Fig. 6). The amorphous exterior was attributed to hydrogenation of the nanoparticle surface layers, which is supported by $\mathrm{Ti}^{3+}$-based ESR signals (Fig. 7)

\section{Photodegradation efficiency}

Rhodamine $\mathrm{B}(\mathrm{RhB})$ was used as a model pollutant to test the photocatalytic activity of black $\mathrm{Nb}_{\mathbf{x}} \mathrm{O}_{\mathrm{y}}-\mathrm{TiO}_{2}$ under simulated sunlight and monochromatic NIR light. The interaction between the hydrophobic surface of black $\mathrm{Nb}_{x} \mathrm{O}_{y}-\mathrm{TiO}_{2}$ and the hydrophobic region of $\mathrm{RhB}$ was supported by Raman spectroscopy, i.e. the xanthene ring and $\mathrm{C}=\mathrm{C}$ Raman shifts at 705 and $1642 \mathrm{~cm}^{-1}$, respectively (Fig. 8). An additional Nb-N stretching band indicates additional covalent binding between $\mathrm{RhB}$ and the $\mathrm{Nb}_{2} \mathrm{O}_{5}$ surface. RhB adsorption also narrows the $\mathrm{Ti}^{3+}$ band in the EPR spectrum.

Black $\mathrm{Nb}_{x} \mathrm{O}_{y}-\mathrm{TiO}_{2}$ displayed superior RhB photodegradation efficiency under NIR and SSL light when compared to black $\mathrm{TiO}_{2}$ (80 and 25\% photodegradation efficiency, respectively under NIR light); however, the difference using SSL light was much less than when using NIR illumination. Photobleaching and photodegradation (as measured via residual TOC) by $\mathrm{Nb}_{x} \mathrm{O}_{y}-\mathrm{TiO}_{2}$ under NIR light followed the same trends (Figs. 9 and 10), and the material is highly stable over 5 reaction cycles (Fig. 11).

\section{Photoactivation mechanism}

The photodegradation mechanism involves the photogeneration of reactive oxygen species (ROS) generated after the formation of photogenerated electron-hole pairs and their diffusion to the catalyst surface. To identify which ROS were involved we used the conversion of TMP-OH to TEMPO-OH, which is EPR active. This reaction is unselective, and any number of ROS will convert TMP-OH to TEMPO-OH. However, using a series of selective ROS scavengers allows for the identification of important ROS through a process of elimination. Using efficient scavengers, i.e., with a high reaction rate constant, at sufficiently high concentration should eliminate all TEMPO-OH formation. Herein $\mathrm{NaN}_{3}, \mathrm{XTT}$, and TBA are used as scavengers of singlet oxygen, superoxide radical anion, and hydroxyl radical, respectively (Fig. 12). $\mathrm{NaN}_{3}$ and XTT quench TEMPO-OH formation, suggesting that superoxide radical anion is formed. $\mathrm{NaN}_{3}$ quenching could indicate the participation of singlet oxygen, but because it does not scavenge superoxide radical 
anion and no TEMPO-OH is formed it is likely acting as a potent hole scavenger as part of an upstream process in superoxide radical anion formation.

9,10-anthracenediylbis(methylene)dimalonic acid (ABDA) was used as a sensor for experiments designed to probe the effects of charge carrier generation. Irradiation of $\mathrm{ABDA}$ and $\mathrm{Nb}_{\mathrm{x}} \mathrm{O}_{\mathrm{y}}-\mathrm{TiO}_{2}$ with an IR laser resulted in complete photobleaching within $30 \mathrm{~min}$ (Fig. 13); however, the addition of oxalate, an efficient hole scavenger, decreased photobleaching by $75 \%$ at the same time. The remaining surface electrons could reduce dissolved oxygen to superoxide radical anions, which could bleach ABDA through either reduction or oxidation. However, sparging the solution with argon or coating the nanoparticles in $\mathrm{SiO}_{2}$ layer prevents ABDA photobleaching altogether (Fig. 13). Because only $25 \%$ of the photobleaching was mediated by surface electrons, $75 \%$ must be mediated by holes. Often holes generate hydroxyl radicals via water oxidation; however, the formation of TEMPO from TMP in the presence of TBA, an efficient hydroxyl radical scavenger, suggests that direct hole-based oxidation may occur.

\section{Upconversion}

$\mathrm{bTiO}_{2}$ has some intrinsic upconversion activity as exemplified by photoluminescence and photoelectrochemical measurements, the intensities of which are greatly enhanced by the presence of the niobium oxide shell. Upon excitation at $785 \mathrm{~nm}\left(30 \mathrm{~mW} \mathrm{~cm}^{-2}\right)$, most light is re-emitted in that envelope, but with significant emission upconverted to $625 \mathrm{~nm}$ (Fig 14). This is more apparent with $15 \mathrm{wt} \% \mathrm{Nb}$ loading. Additionally, in photoelectrochemical measurements, i.e., the I-V characteristic (Fig. 15), shows no photocurrent generated in the dark or by white $\mathrm{TiO}_{2}$, but upon excitation of $\mathrm{bTiO}_{2}$ at $655 \mathrm{~nm}(30 \mathrm{~mW} \mathrm{~cm}$

$\left.{ }^{2}\right)$ there is a distinct photocurrent visible. Similar results are observed upon excitation at $785 \mathrm{~nm}$, but the enhancement in photocurrent induced by the addition of niobium oxide layers is dramatic.

\section{Conclusion}

Black $\mathrm{Nb}_{x} \mathrm{O}_{\mathrm{y}}-\mathrm{TiO}_{2}$ nanoparticles have broad light adsorption into the infrared region, enabling efficient solar spectrum and NIR light utilization compared with contemporary black $\mathrm{TiO}_{2}$ materials through enhanced charge transfer enabled by $\mathrm{Nb}_{x} \mathrm{O}_{y}$ phases, increased conductivity, and rapid exciton splitting, and enhanced upconversion. Photobleaching of dyes appears to be dominated by direct hole oxidation with significant participation of superoxide radical anion formation. This material has potential applications in IR imaging, photochemical/thermal treatment of cancer, and antibiotic and anti-biofouling surfaces.

\section{References}

1. Björnsdotter, M. K.; Yeung, L. W. Y.; Kärrman, A.; Jogsten, I. E., Ultra-Short-Chain Perfluoroalkyl Acids Including Trifluoromethane Sulfonic Acid in Water Connected to Known and Suspected Point Sources in Sweden. Environmental Science \& Technology 2019, 53 (19), 11093-11101.

2. Stets, E. G.; Sprague, L. A.; Oelsner, G. P.; Johnson, H. M.; Murphy, J. C.; Ryberg, K.; Vecchia, A. V.; Zuellig, R. E.; Falcone, J. A.; Riskin, M. L., Landscape Drivers of Dynamic Change in Water Quality of U.S. Rivers. Environmental Science \& Technology 2020, 54 (7), 4336-4343.

3. Coulter, D. P.; Huff Hartz, K. E.; Sepúlveda, M. S.; Godfrey, A.; Garvey, J. E.; Lydy, M. J., Lifelong Exposure to Dioxin-Like PCBs Alters Paternal Offspring Care Behavior and Reduces Male Fish Reproductive Success. Environmental Science \& Technology 2019, 53 (19), 11507-11514.

4. Wang, Z.; Mi, B., Environmental Applications of 2D Molybdenum Disulfide (MoS2) Nanosheets. Environmental Science \& Technology 2017, 51 (15), 8229-8244. 
5. Routoula, E.; Patwardhan, S. V., Degradation of Anthraquinone Dyes from Effluents: A Review Focusing on Enzymatic Dye Degradation with Industrial Potential. Environmental Science \& Technology 2020, 54 (2), 647-664.

6. Chu, C.; Yang, J.; Huang, D.; Li, J.; Wang, A.; Alvarez, P. J. J.; Kim, J.-H., Cooperative Pollutant Adsorption and Persulfate-Driven Oxidation on Hierarchically Ordered Porous Carbon. Environmental Science \& Technology 2019, 53 (17), 10352-10360.

7. Hanhauser, E.; Bono, M. S.; Vaishnav, C.; Hart, A. J.; Karnik, R., Solid-Phase Extraction, Preservation, Storage, Transport, and Analysis of Trace Contaminants for Water Quality Monitoring of Heavy Metals. Environmental Science \& Technology 2020, 54 (5), 2646-2657.

8. Ge, Q.; Feng, X.; Wang, R.; Zheng, R.; Luo, S.; Duan, L.; Ji, Y.; Lin, J.; Chen, H., Mixed RedoxCouple-Involved Chalcopyrite Phase CuFeS2 Quantum Dots for Highly Efficient Cr(VI) Removal. Environmental Science \& Technology 2020, 54 (13), 8022-8031.

9. $\quad$ Fan, Y. Z.; Han, L.; Yang, Y. Z.; Sun, Z.; Li, N.; Li, B. L.; Luo, H. Q.; Li, N. B., Multifunctional Binding Strategy on Nonconjugated Polymer Nanoparticles for Ratiometric Detection and Effective Removal of Mercury lons. Environmental Science \& Technology 2020.

10. El-Morsi, T. M.; Budakowski, W. R.; Abd-El-Aziz, A. S.; Friesen, K. J., Photocatalytic Degradation of 1,10-Dichlorodecane in Aqueous Suspensions of TiO2: A Reaction of Adsorbed Chlorinated Alkane with Surface Hydroxyl Radicals. Environmental Science \& Technology 2000, 34 (6), 1018-1022.

11. Liu, C.; Kong, D.; Hsu, P.-C.; Yuan, H.; Lee, H.-W.; Liu, Y.; Wang, H.; Wang, S.; Yan, K.; Lin, D.; Maraccini, P. A.; Parker, K. M.; Boehm, A. B.; Cui, Y., Rapid water disinfection using vertically aligned MoS2 nanofilms and visible light. Nature Nanotechnology 2016, 11 (12), 1098-1104.

12. Gao, Y.; Wu, J.; Wang, J.; Fan, Y.; Zhang, S.; Dai, W., A Novel Multifunctional p-Type Semiconductor@MOFs Nanoporous Platform for Simultaneous Sensing and Photodegradation of Tetracycline. ACS Applied Materials \& Interfaces 2020, 12 (9), 11036-11044.

13. Kaur, M.; Shinde, S. L.; Ishii, S.; Jevasuwan, W.; Fukata, N.; Yu, M.-W.; Li, Y.; Ye, J.; Nagao, T., Marimo-Bead-Supported Core-Shell Nanocomposites of Titanium Nitride and Chromium-Doped Titanium Dioxide as a Highly Efficient Water-Floatable Green Photocatalyst. ACS Applied Materials \& Interfaces 2020, 12 (28), 31327-31339.

14. Mavuso, M. A.; Makgwane, P. R.; Ray, S. S., Heterostructured CeO2-M (M = Co, Cu, Mn, Fe, Ni) Oxide Nanocatalysts for the Visible-Light Photooxidation of Pinene to Aroma Oxygenates. ACS Omega 2020, 5 (17), 9775-9788.

15. Lee, S. Y.; Kang, D.; Jeong, S.; Do, H. T.; Kim, J. H., Photocatalytic Degradation of Rhodamine B Dye by $\mathrm{TiO} 2$ and Gold Nanoparticles Supported on a Floating Porous Polydimethylsiloxane Sponge under Ultraviolet and Visible Light Irradiation. ACS Omega 2020, 5 (8), 4233-4241.

16. Chen, H.; Nanayakkara, C. E.; Grassian, V. H., Titanium Dioxide Photocatalysis in Atmospheric Chemistry. Chemical Reviews 2012, 112 (11), 5919-5948.

17. Sang, L.; Zhao, Y.; Burda, C., TiO2 Nanoparticles as Functional Building Blocks. Chemical Reviews 2014, 114 (19), 9283-9318.

18. Liu, K.; Cao, M.; Fujishima, A.; Jiang, L., Bio-Inspired Titanium Dioxide Materials with Special Wettability and Their Applications. Chemical Reviews 2014, 114 (19), 10044-10094.

19. Chen, X.; Liu, L.; Yu, P. Y.; Mao, S. S., Increasing Solar Absorption for Photocatalysis with Black Hydrogenated Titanium Dioxide Nanocrystals. Science 2011, 331 (6018), 746.

20. Kar, A.; Smith, Y. R.; Subramanian, V., Improved Photocatalytic Degradation of Textile Dye Using Titanium Dioxide Nanotubes Formed Over Titanium Wires. Environmental Science \& Technology 2009, 43 (9), 3260-3265.

21. Chen, X.; Liu, L.; Huang, F., Black titanium dioxide (TiO2) nanomaterials. Chemical Society Reviews 2015, 44 (7), 1861-1885. 
22. Liu, G.; Yang, H. G.; Pan, J.; Yang, Y. Q.; Lu, G. Q.; Cheng, H.-M., Titanium Dioxide Crystals with Tailored Facets. Chemical Reviews 2014, 114 (19), 9559-9612.

23. Xie, L.; Zhu, Q.; Zhang, G.; Ye, K.; Zou, C.; Prezhdo, O. V.; Wang, Z.; Luo, Y.; Jiang, J., Tunable Hydrogen Doping of Metal Oxide Semiconductors with Acid-Metal Treatment at Ambient Conditions. Journal of the American Chemical Society 2020, 142 (9), 4136-4140.

24. Nie, Z.; Ke, X.; Li, D.; Zhao, Y.; Zhu, L.; Qiao, R.; Zhang, X. L., NaYF4:Yb,Er,Nd@NaYF4:Nd Upconversion Nanocrystals Capped with Mn:TiO2 for 808 nm NIR-Triggered Photocatalytic Applications. The Journal of Physical Chemistry C 2019, 123 (37), 22959-22970.

25. Li, M.; Zheng, Z.; Zheng, Y.; Cui, C.; Li, C.; Li, Z., Controlled Growth of Metal-Organic Framework on Upconversion Nanocrystals for NIR-Enhanced Photocatalysis. ACS Applied Materials \& Interfaces 2017, 9 (3), 2899-2905.

26. Tan, L.; Li, D.; Zhang, L.; Xu, L.; Zhao, Y.; Zhu, L.; Qiao, R., Preparation of Multishell-Structured NaYF4:Yb,Tm,Nd@NaYF4:Yb,Nd@SiO2@ZnO Nanospheres with Effective NIR-Induced Photocatalytic Activity. The Journal of Physical Chemistry C 2020.

27. Zheng, W.; Huang, P.; Gong, Z.; Tu, D.; Xu, J.; Zou, Q.; Li, R.; You, W.; Bünzli, J.-C. G.; Chen, X., Near-infrared-triggered photon upconversion tuning in all-inorganic cesium lead halide perovskite quantum dots. Nature Communications 2018, 9 (1), 3462.

28. Xie, L.; Lin, C.; Chen, Q.; Yang, H.-H., 14 - Upconversion Nanomaterials for Near-infrared LightMediated Theranostics. In Theranostic Bionanomaterials, Cui, W.; Zhao, X., Eds. Elsevier: 2019; pp 321-340.

29. Wang, Y.; Deng, R.; Xie, X.; Huang, L.; Liu, X., Nonlinear spectral and lifetime management in upconversion nanoparticles by controlling energy distribution. Nanoscale 2016, 8 (12), 6666-6673.

30. Auzel, F., Upconversion and Anti-Stokes Processes with $\mathrm{f}$ and $\mathrm{d}$ Ions in Solids. Chemical Reviews 2004, 104 (1), 139-174.

31. Naldoni, A.; Altomare, M.; Zoppellaro, G.; Liu, N.; Kment, Š.; Zbořil, R.; Schmuki, P., Photocatalysis with Reduced TiO2: From Black TiO2 to Cocatalyst-Free Hydrogen Production. ACS Catalysis 2019, 9 (1), 345-364.

32. Pinto, M. B.; Soares, A. L.; Quintão, M. C.; Duarte, H. A.; De Abreu, H. A., Unveiling the Structural and Electronic Properties of the B-Nb2O5 Surfaces and Their Interaction with $\mathrm{H} 2 \mathrm{O}$ and $\mathrm{H} 2 \mathrm{O} 2$. The Journal of Physical Chemistry C 2018, 122 (12), 6618-6628.

33. Foo, G. S.; Wei, D.; Sholl, D. S.; Sievers, C., Role of Lewis and Brønsted Acid Sites in the Dehydration of Glycerol over Niobia. ACS Catalysis 2014, 4 (9), 3180-3192.

34. Bolzan, A. A.; Fong, C.; Kennedy, B. J.; Howard, C. J., A Powder Neutron Diffraction Study of Semiconducting and Metallic Niobium Dioxide. Journal of Solid State Chemistry 1994, 113 (1), 9-14.

35. Hadamek, T.; Posadas, A. B.; Dhamdhere, A.; Smith, D. J.; Demkov, A. A., Spectral identification scheme for epitaxially grown single-phase niobium dioxide. Journal of Applied Physics 2016, 119 (9), 095308.

36. O'Hara, A.; Nunley, T. N.; Posadas, A. B.; Zollner, S.; Demkov, A. A., Electronic and optical properties of NbO2. Journal of Applied Physics 2014, 116 (21), 213705.

37. Penn, R. L.; Banfield, J. F., Formation of rutile nuclei at anatase $\{112\}$ twin interfaces and the phase transformation mechanism in nanocrystalline titania American Mineralogist 1999, 84 (5-6).

38. Tian, M.; Mahjouri-Samani, M.; Eres, G.; Sachan, R.; Yoon, M.; Chisholm, M. F.; Wang, K.; Puretzky, A. A.; Rouleau, C. M.; Geohegan, D. B.; Duscher, G., Structure and Formation Mechanism of Black TiO2 Nanoparticles. ACS Nano 2015, 9 (10), 10482-10488.

39. Subedi, B.; Du, B.; Chambliss, C. K.; Koschorreck, J.; Rüdel, H.; Quack, M.; Brooks, B. W.; Usenko, S., Occurrence of Pharmaceuticals and Personal Care Products in German Fish Tissue: A National Study. Environmental Science \& Technology 2012, 46 (16), 9047-9054.

40. Nosaka, Y.; Nosaka, A. Y., Generation and Detection of Reactive Oxygen Species in Photocatalysis. Chemical Reviews 2017, 117 (17), 11302-11336. 
41. De Trizio, L.; Buonsanti, R.; Schimpf, A. M.; Llordes, A.; Gamelin, D. R.; Simonutti, R.; Milliron, D. J., Nb-Doped Colloidal TiO2 Nanocrystals with Tunable Infrared Absorption. Chemistry of Materials 2013, 25 (16), 3383-3390.

42. Yang, X.; Zhang, M.-J.; Min, Y.; Xu, M.; Mei, Z.; Liang, J.; Hu, J.; Yuan, S.; Xiao, S.; Duan, Y.; Liu, F.; Lin, H.; Lin, Y.; Pan, F., Controllable Formation of (004)-Orientated Nb:TiO2 for High-Performance Transparent Conductive Oxide Thin Films with Tunable Near-Infrared Transmittance. ACS Applied Materials \& Interfaces 2017, 9 (34), 29021-29029. 
Figures

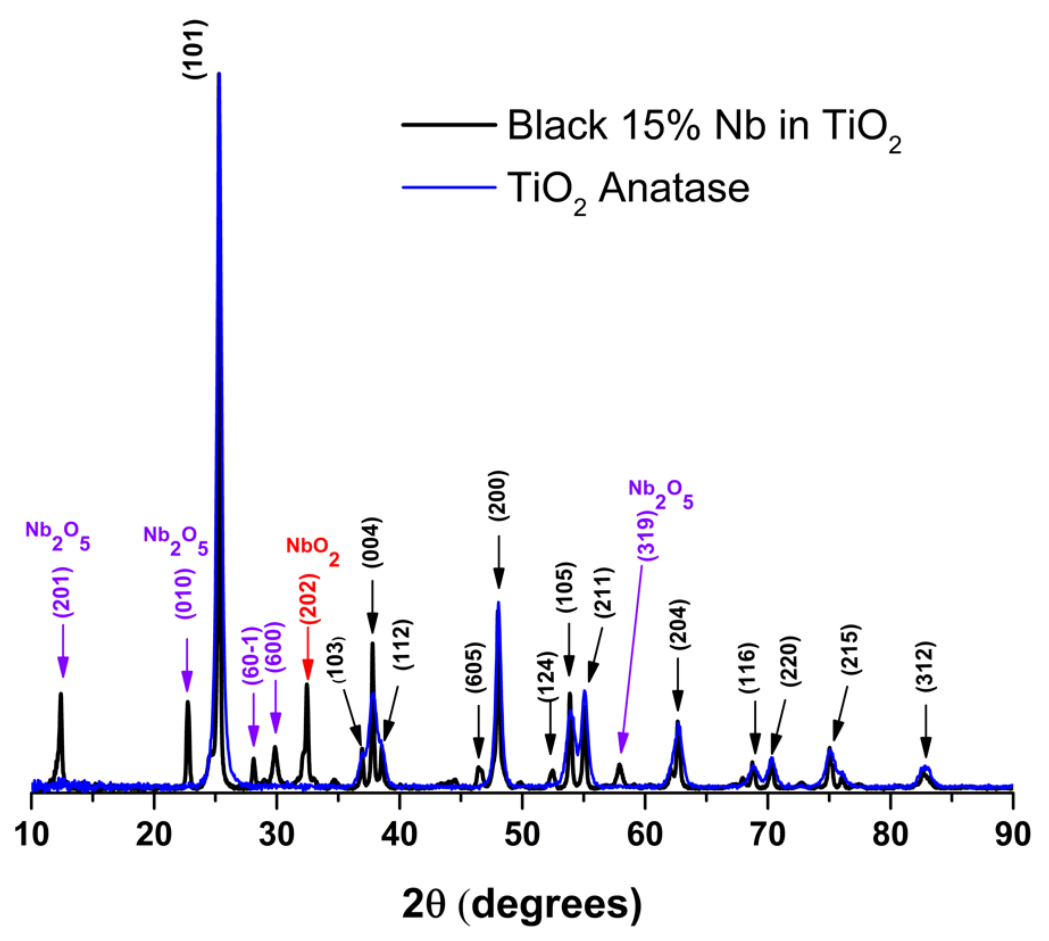

Fig. 1. Powder $\mathrm{X}$-ray diffraction patterns for anatase and black $\mathrm{Nb}_{x} \mathrm{O}_{\mathrm{y}}-\mathrm{TiO}_{2}$ nanoparticles.

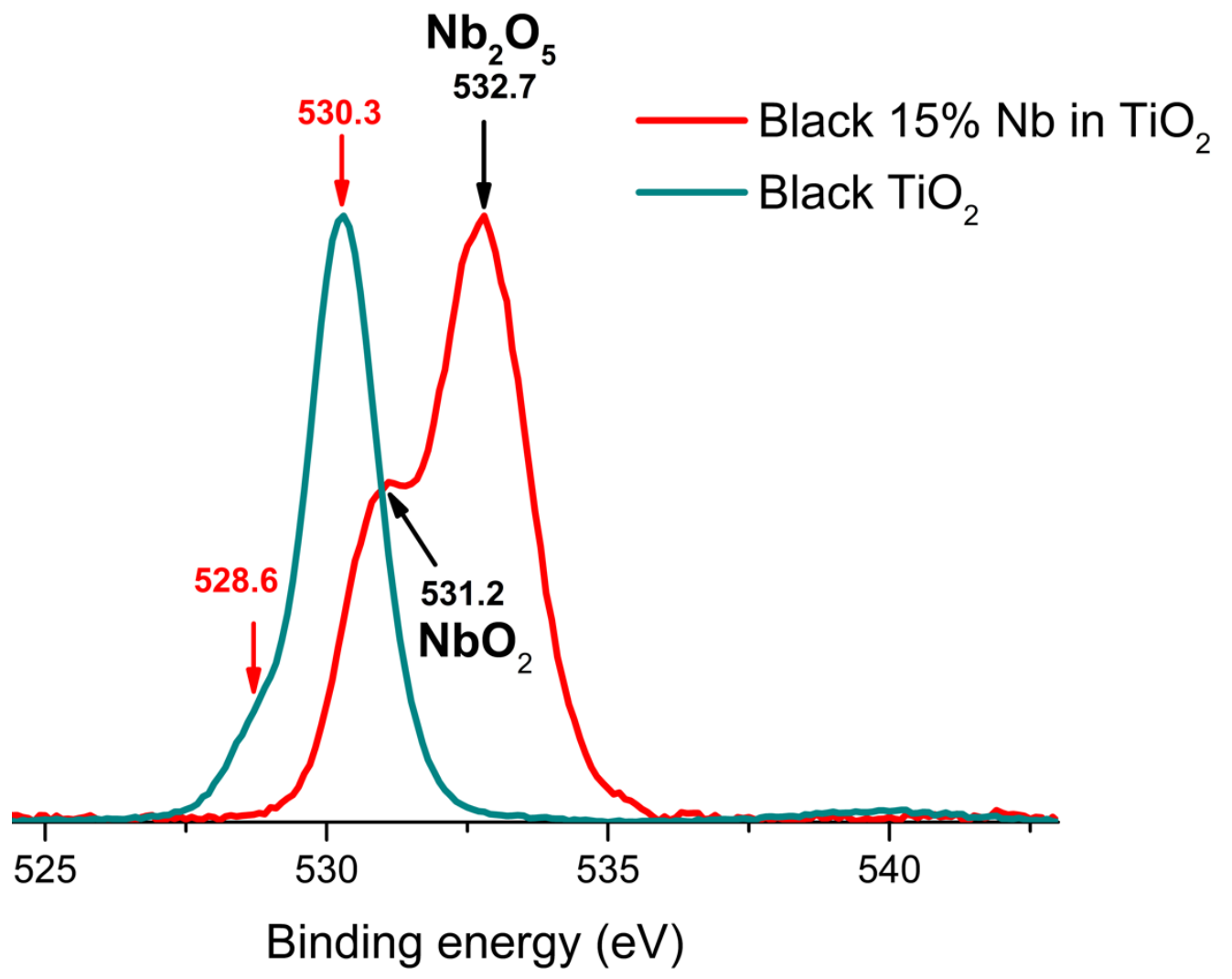

Fig. 2. O1s X-ray photoelectron spectra for black $\mathrm{TiO}_{2}$ and black $\mathrm{Nb}_{x} \mathrm{O}_{y}-\mathrm{TiO}_{2}$ nanoparticles. 


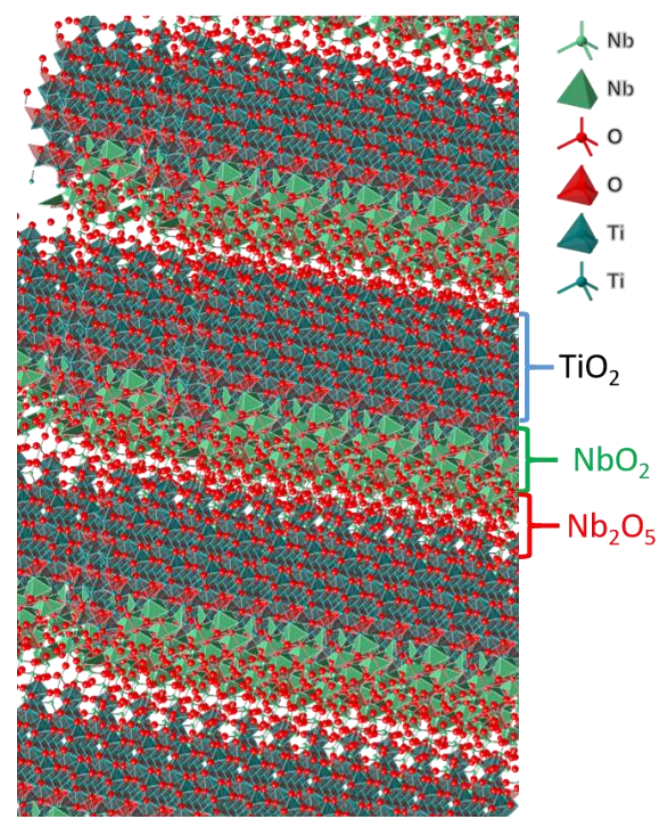

Fig. 3. The proposed supercell for $\mathrm{Nb}_{x} \mathrm{O}_{\mathrm{y}}-\mathrm{TiO}_{2}$ nanoparticles.

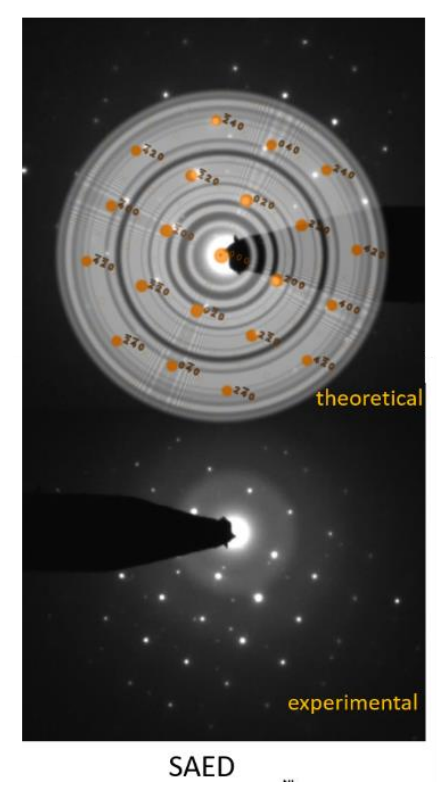

Fig. 4. Theoretical and observed selected area electron diffraction patterns for $\mathrm{Nb}_{x} \mathrm{O}_{y}-\mathrm{TiO}_{2}$ nanoparticles. 


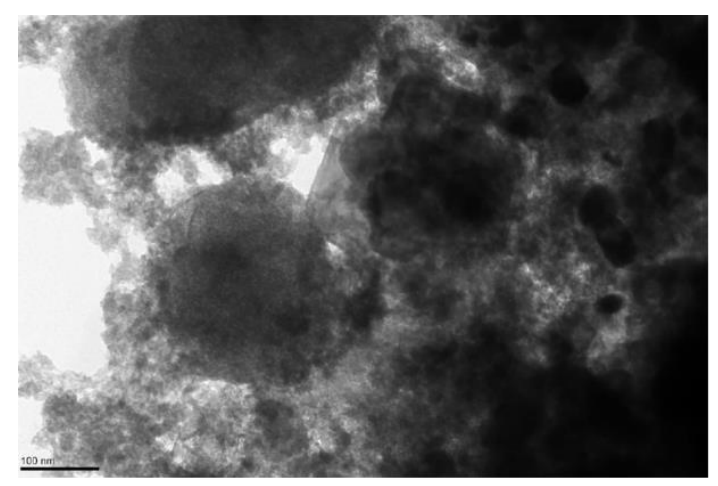

Fig. 5. Transmission electron micrograph of spheroidal black $\mathrm{Nb}_{x} \mathrm{O}_{y}-\mathrm{TiO}_{2}$ nanoparticles.

\section{Hydrogenated}

Amorphous layer

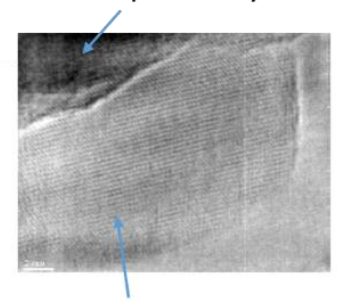

Crystal layer

Fig. 6. High resolution transmission electron micrograph of a black $\mathrm{Nb}_{x} \mathrm{O}_{y}-\mathrm{TiO}_{2}$ nanoparticle showing a hydrogenated amorphous layer.

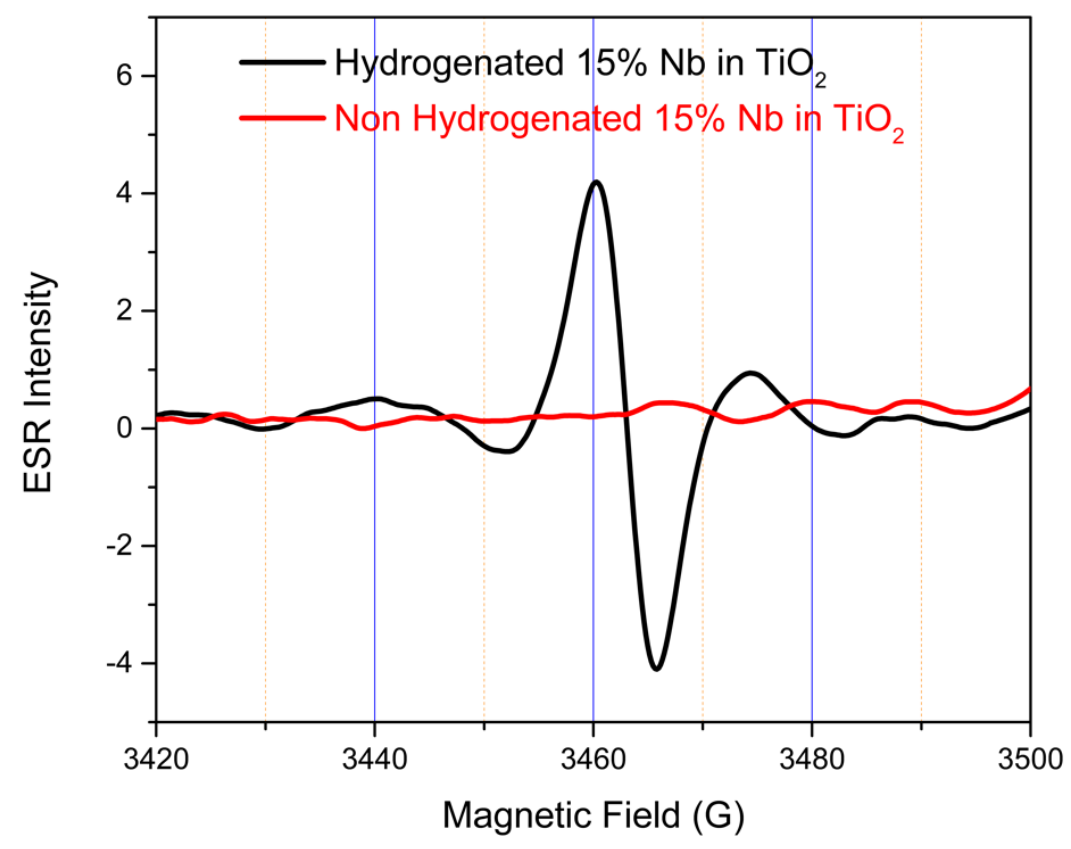

Fig. 7. Electron paramagnetic resonance spectra of black and white $\mathrm{Nb}_{x} \mathrm{O}_{y}-\mathrm{TiO}_{2}$ nanoparticles. 


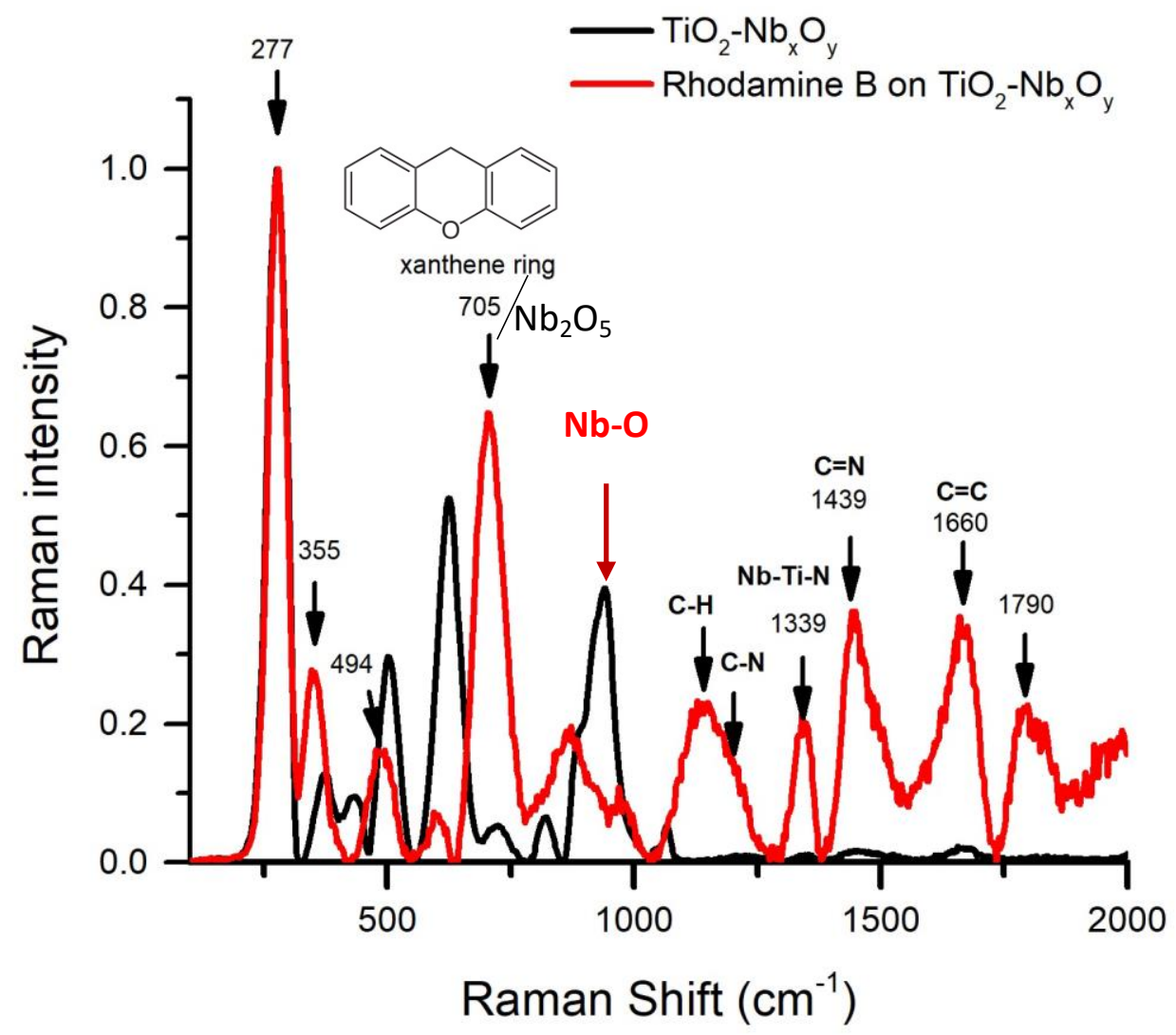

Fig. 8. Raman spectra of black $\mathrm{Nb}_{x} \mathrm{O}_{y}-\mathrm{TiO}_{2}$ nanoparticles with and without adsorbed Rhodamine $\mathrm{B}$.
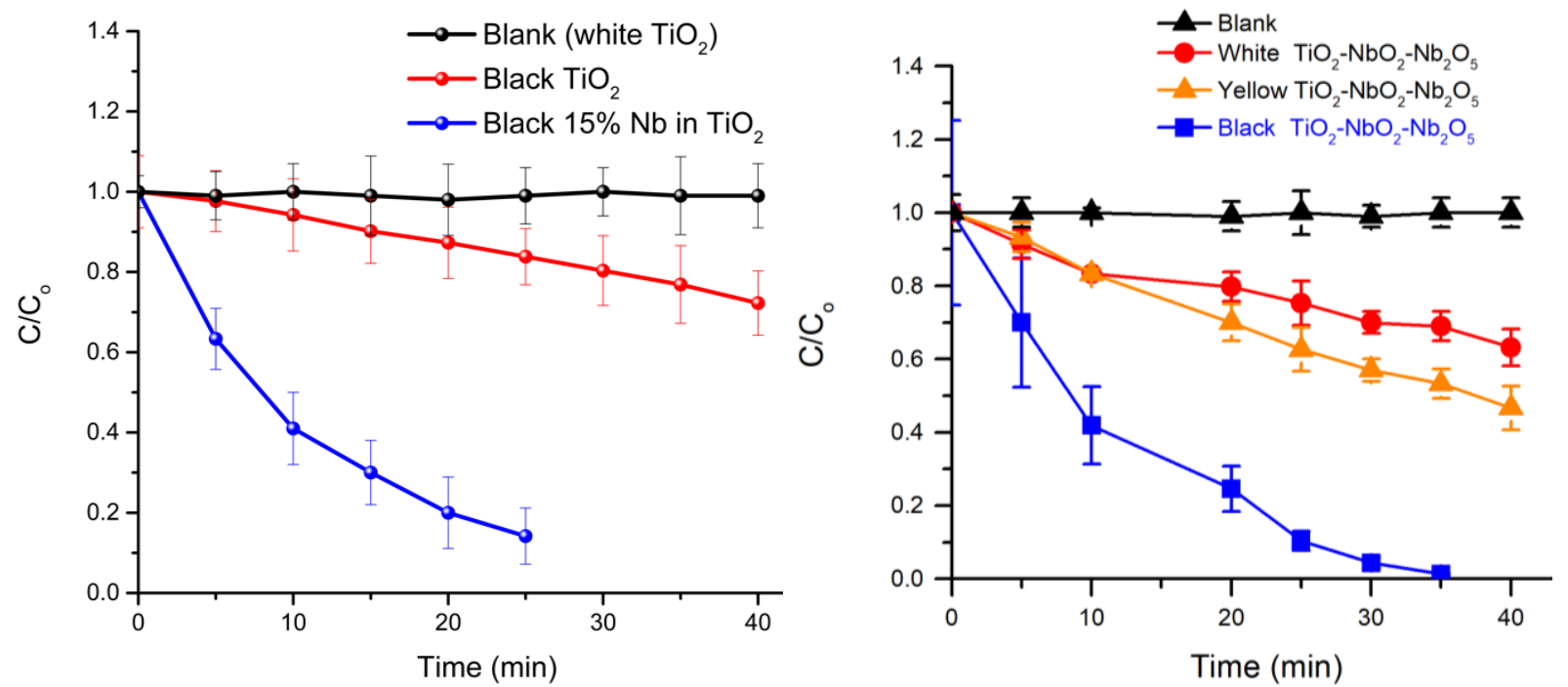

Fig. 9. Photobleaching of Rhodamine B by varying photocatalysts under (left) $830 \mathrm{~nm}$ radiation and (right) simulated sunlight. 

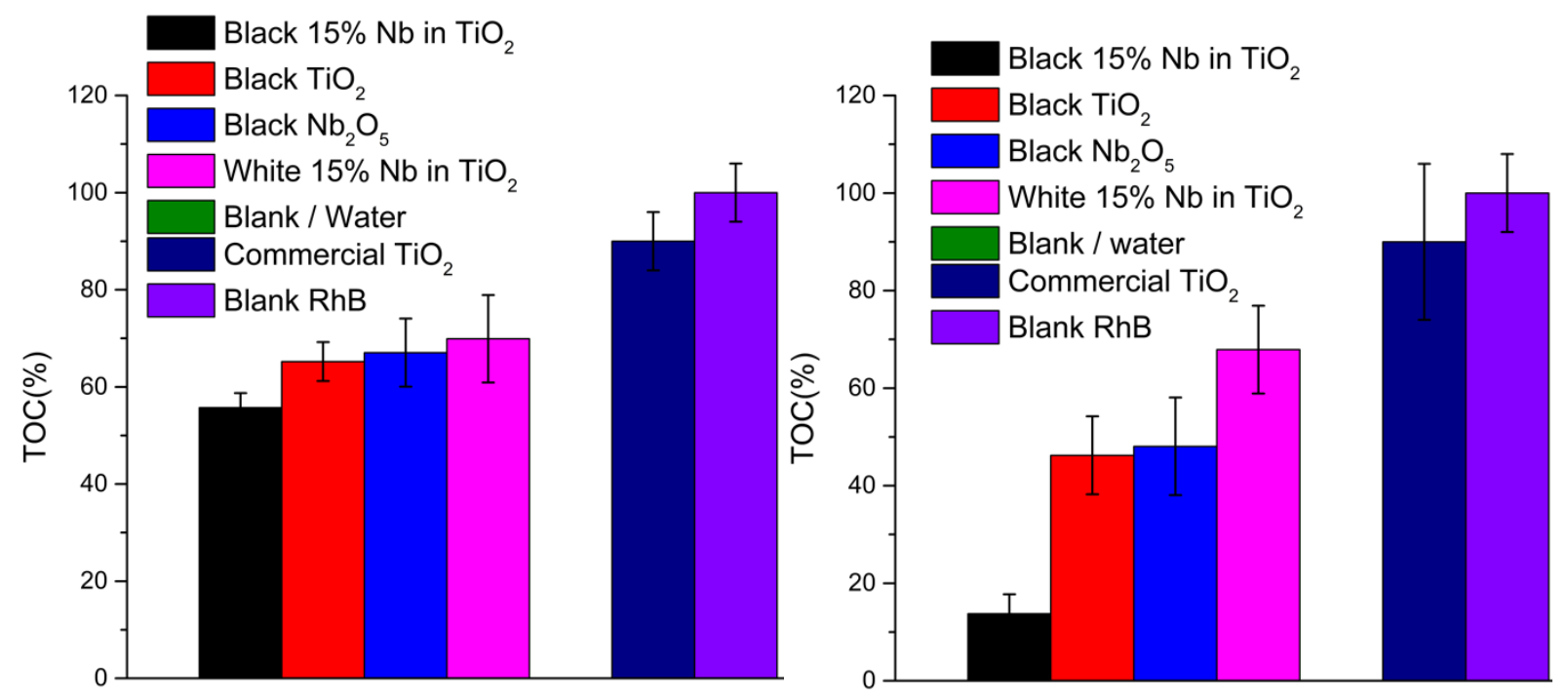

Fig. 10. Photomineralization of Rhodamine B by various photocatalysts. Residual total organic carbon (left) before irradiation (adsorption only) and (right) after irradiation at $830 \mathrm{~nm}$.
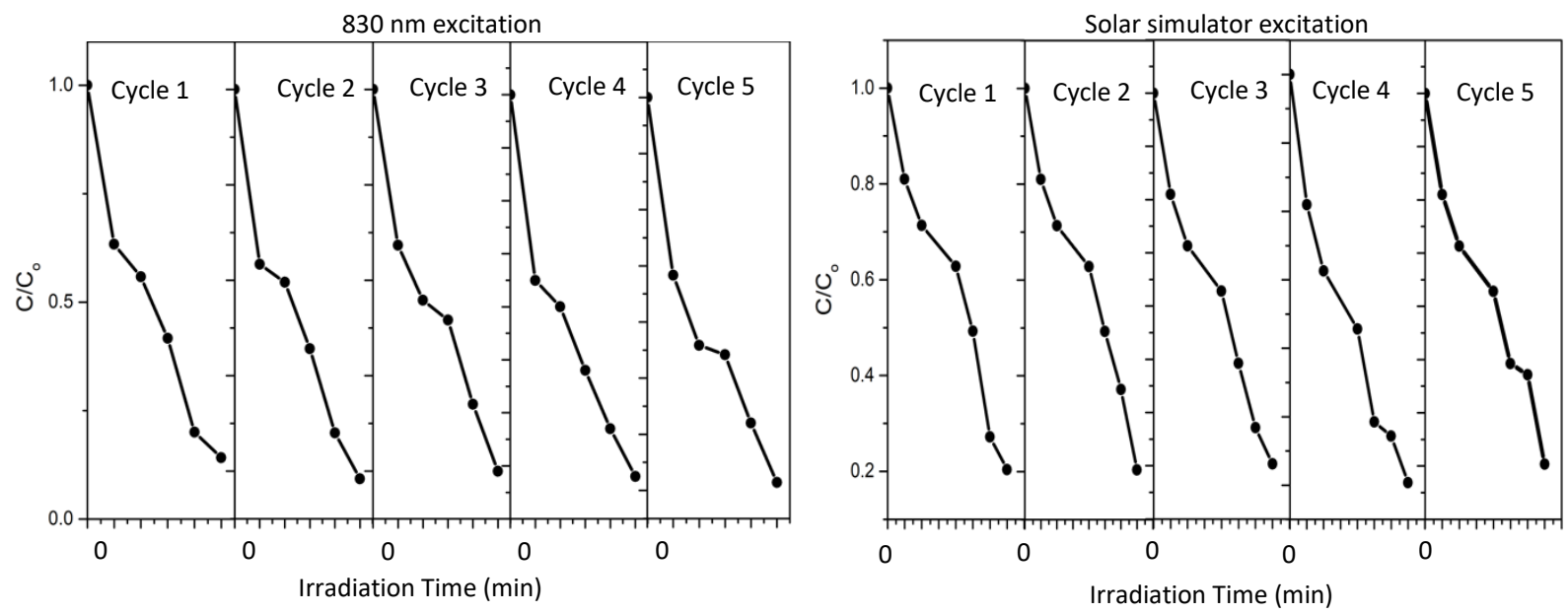

Fig. 11. Photodegradation of Rhodamine $\mathrm{B}$ over several cycles by black $\mathrm{Nb}_{\mathrm{x}} \mathrm{O}_{\mathrm{y}}-\mathrm{TiO}_{2}$ nanoparticles irradiated by (left) $830 \mathrm{~nm}$ light and (right) simulated sunlight. 


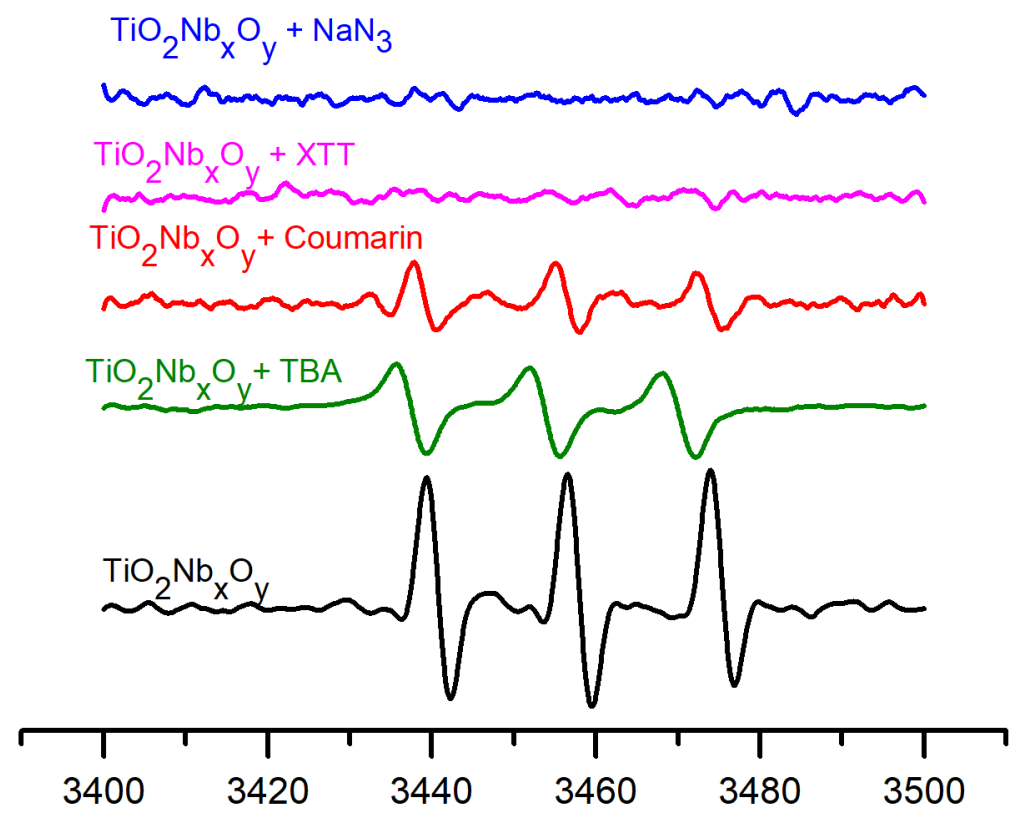

Field (G)

Fig. 12. Electron paramagnetic resonance spectra of 4-hydroxy-2,2,6,6-tetramethylpiperidine after irradiation in the presense of black $\mathrm{Nb}_{x} \mathrm{O}_{y}-\mathrm{TiO}_{2}$ nanoparticles in the presence of various radical scavengers. 


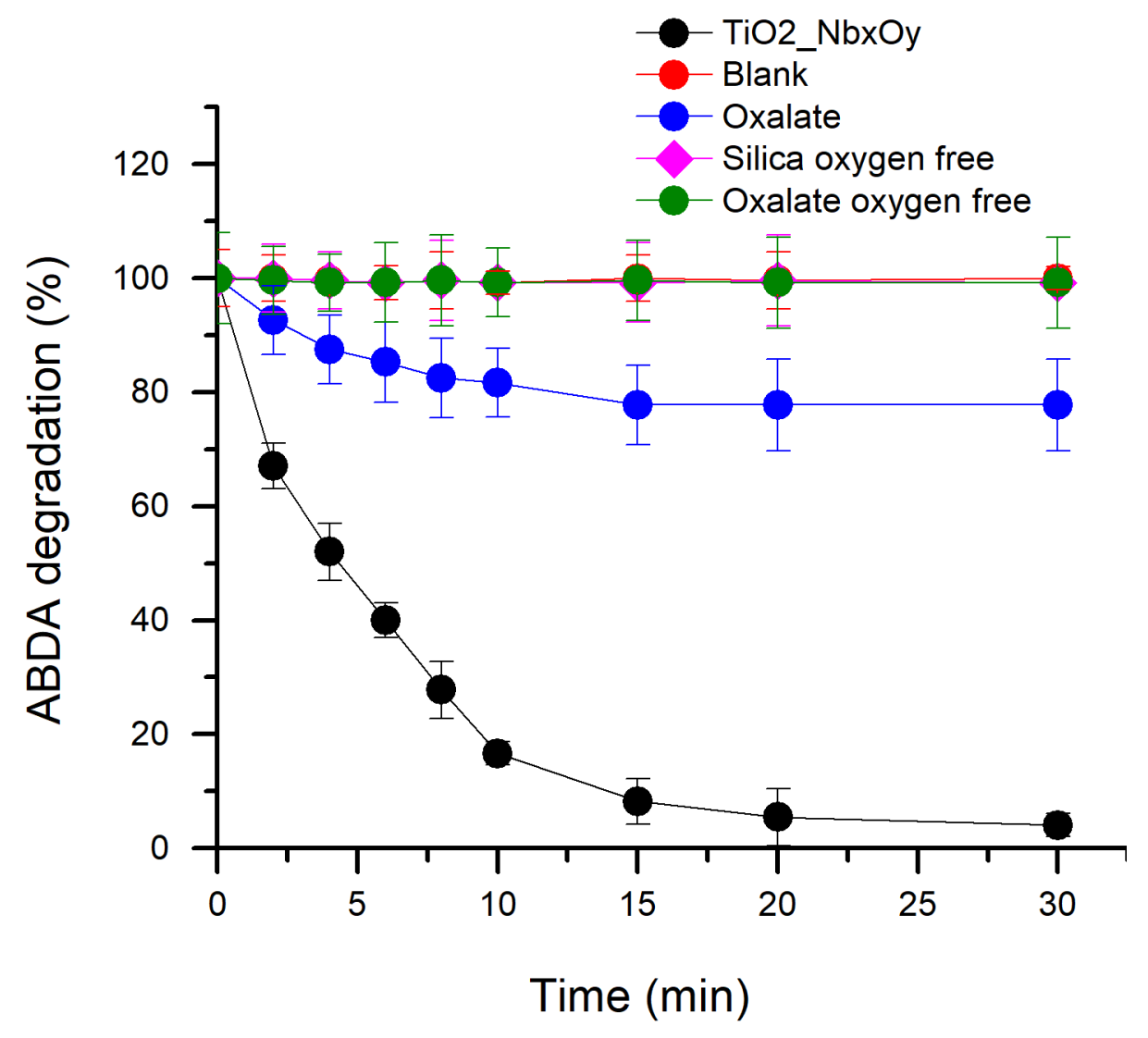

Fig. 13. Photodegradation of $A B D A$ in the presence of black $\mathrm{Nb}_{x} \mathrm{O}_{y}-\mathrm{TiO}_{2}$ nanoparticles with varying scavengers. 


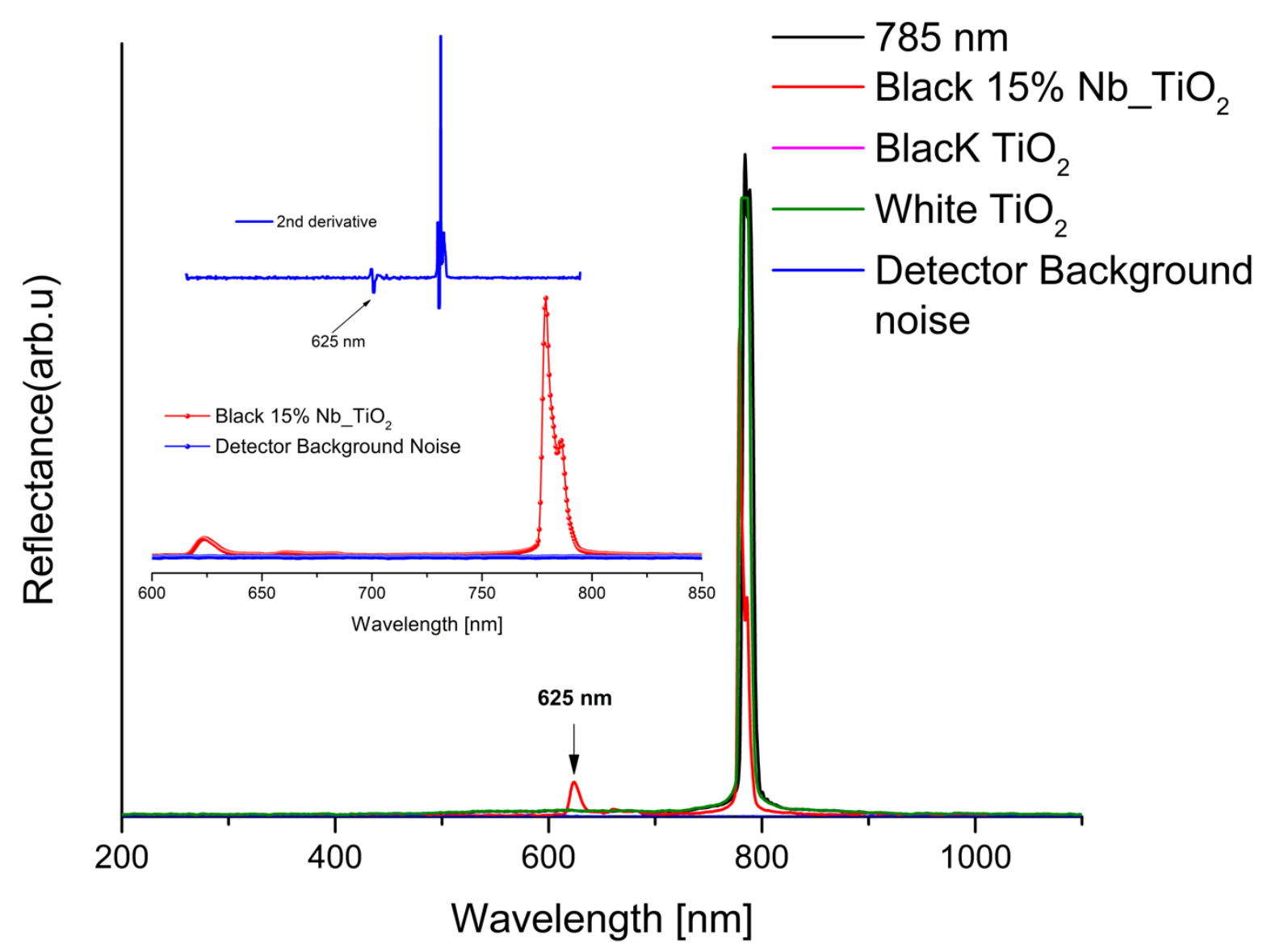

Fig. 14. Photoluminescence experiments using varying photocatalysts (excitation wavelength $=785 \mathrm{~nm}$ ).
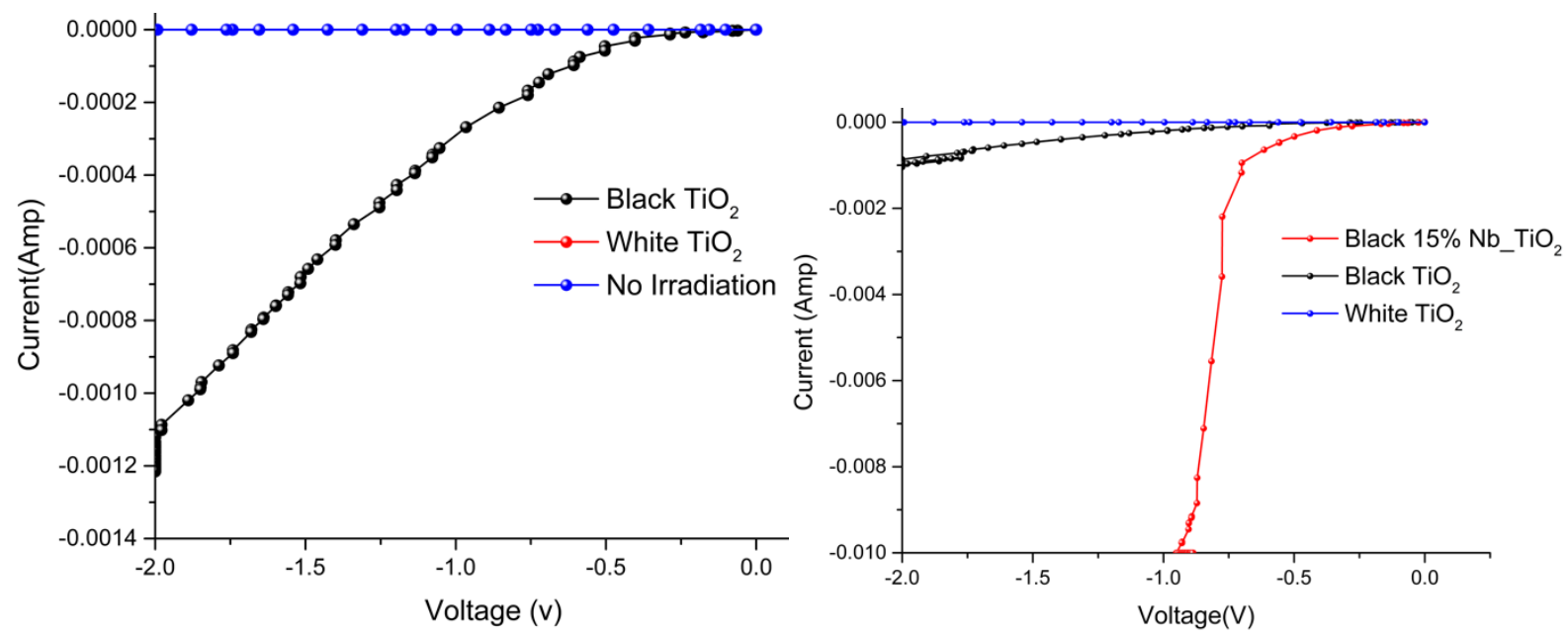

Fig. 15. Photoelectrochemical experiments using varying photocatalysts. I-V characteristic photocurrents generated by (left) $655 \mathrm{~nm}$ and (right) $785 \mathrm{~nm}$ irradiation (30 mW cm${ }^{-2}$ ). 